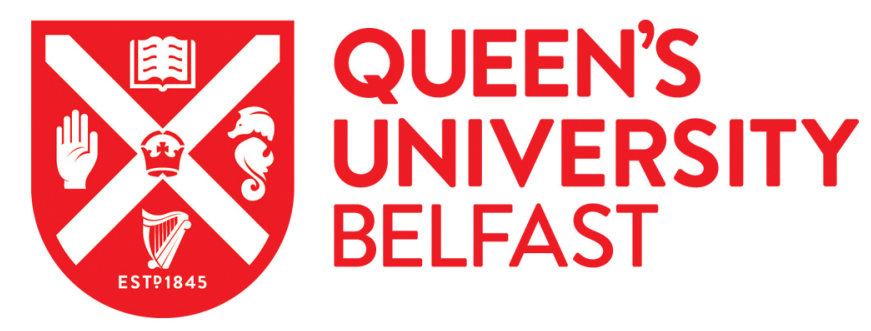

\title{
A Two-Ray Multipath Model for Frequency Diverse Array-Based Directional Modulation in MISOME Wiretap Channels
}

Cheng, Q., Fusco, V., Wang, S., \& Zhu, J. (2019). A Two-Ray Multipath Model for Frequency Diverse ArrayBased Directional Modulation in MISOME Wiretap Channels. In 2019 IEEE 90th Vehicular Technology Conference, VTC 2019 Fall - Proceedings [8890978] (IEEE Vehicular Technology Conference; Vol. 2019September). Institute of Electrical and Electronics Engineers Inc.. https://doi.org/10.1109/VTCFall.2019.8890978

Published in:

2019 IEEE 90th Vehicular Technology Conference, VTC 2019 Fall - Proceedings

Document Version:

Peer reviewed version

Queen's University Belfast - Research Portal:

Link to publication record in Queen's University Belfast Research Portal

Publisher rights

Copyright 2019 IEEE. This work is made available online in accordance with the publisher's policies. Please refer to any applicable terms of use of the publisher.

\section{General rights}

Copyright for the publications made accessible via the Queen's University Belfast Research Portal is retained by the author(s) and / or other copyright owners and it is a condition of accessing these publications that users recognise and abide by the legal requirements associated with these rights.

Take down policy

The Research Portal is Queen's institutional repository that provides access to Queen's research output. Every effort has been made to ensure that content in the Research Portal does not infringe any person's rights, or applicable UK laws. If you discover content in the Research Portal that you believe breaches copyright or violates any law, please contact openaccess@qub.ac.uk. 


\title{
A Two-Ray Multipath Model for Frequency Diverse Array-Based Directional Modulation in MISOME Wiretap Channels
}

\author{
Qian Cheng ${ }^{* \dagger}$, Vincent Fusco ${ }^{\dagger}$, Shilian Wang*, and Jiang Zhu* \\ * College of Electronic Science, National University of Defense Technology, Changsha, China \\ $\dagger$ Institute of Electronics, Communications and Information Technology (ECIT), Queen's University Belfast, Belfast, UK \\ chengqian14a@nudt.edu.cn,v.fusco@ecit.qub.ac.uk, \{wangsl,jiangzhu\}@nudt.edu.cn
}

\begin{abstract}
A two-ray multipath model for frequency diverse array (FDA)-based directional modulation (DM) is proposed in multi-input single-output multi-eavesdropper (MISOME) wiretap channels for the first time. The excitation factors of the FDA and the weight coefficients of the inserted artificial noise (AN) are jointly designed, which impose no impact on the desired receiver while simultaneously distorting the received signals of eavesdroppers. Secrecy rate is analyzed for the proposed two-ray multipath FDA-based DM model. Numerical simulations verified the capability of physical layer secure (PLS) transmissions of the proposed FDA-DM model in two-ray multipath MISOME wiretap channels.
\end{abstract}

Index Terms-Directional modulation; physical layer security; frequency diverse array; multi-path; secrecy rate; MISOME.

\section{INTRODUCTION}

Compared with the conventional phased arrays (PA)-based directional modulation (DM) technology [1][2], the frequency diverse arrays (FDA)-based DM scheme is a newly proposed technology with the advantage of wireless physical layer secure (PLS) transmissions in not only angle dimension but also range dimension.

The FDA technology was first proposed in [3], which applied a small frequency increment across the antenna elements and thus produced a range-angle-dependent beampattern. Afterwards, the work in [4] divided the FDA elements into multiple subarrays and optimized the transmit beamspace matrix with convex optimization for the purpose of range and angle estimations of targets. An improved FDA radar with logarithmically increasing frequency increments was generalized in [5], which obtained a beampattern with a single maximum at the target location. Multiple carriers were combined with FDA in [6] to generate a more focusing dotshaped beampattern. The authors in [7] put forward the random frequency diverse array for uncoupled range-angle indication in active sensing. A focused beampattern synthesis was put forward in [8] by optimizing the frequency increments with the genetic algorithm, where both single-dot and multidot-shaped transmit beampatterns can be synthesized. Recently, the work in [9] reviewed the developments of the FDA technology.

Owing to the favorable characteristic that the beampattern is both range and angle dependent, the FDA technology has been applied into DM synthesis for wireless PLS transmissions in recent years [10]-[16]. Specifically, the authors in
[10] achieved DM synthesis using the FDA with linearly increasing frequency increments. The FDA with symmetrically and non-linearly increasing frequency increments was used in [11] to decouple range and angle dependent transmit beampattern for wireless DM transmissions. The work in [12] explored the FDA with random frequency increments for DM transmissions. The time-modulated FDA was utilized in [13] to perform wireless PLS transmissions in free space. Additionally, the work in [14] showed that the FDA-based DM is still achievable in Nakagami- $m$ fading channels. The spread spectrum technology was combined with orthogonal FDA in [15] to achieve multi-beam DM synthesis in free space. Recently, the work in [16] generalized the wireless PLS transmissions based on FDA technology for proximal legitimate user and eavesdropper.

The above-mentioned FDA-based DM schemes [10]-[16], however, mainly focused on wireless PLS transmissions in single-path environments without considering the multipath effects. Although some works explored the multipath characteristics of the FDA, such as [17], they did not provide any investigations about the possibility of applying the FDA into DM transmissions in such multipath environments. To the best of our knowledge, no work is available in the present literature with respect to the capability of wireless PLS transmissions of the FDA-based DM technology in multipath environments. This paper is dedicated, for the first time, to extending the FDA-based DM scheme into multipath environments by proposing a simplified two-ray multipath FDA-DM model in multi-input single-output multi-eavesdropper (MISOME) wiretap channels.

The rest of this paper is organized as follows. Section II elaborates the main principles of the proposed two-ray multipath FDA-DM model in MISOME wiretap channels. The secrecy rate of the proposed two-ray multipath FDA-DM model is analyzed in Section III. Simulations about bit error rate (BER) and secrecy rate are provided in Section IV. Finally, Section V makes a conclusion for the whole paper.

Notations: Throughout the paper, scalars and vectors are denoted by italic lower-case letters and arrowed bold lowercase letters, respectively. The operator "." stands for the product of two vectors. $\mathbb{E}(*)$ means the expectation operation of a random variable, while $\mathcal{C N}\left(0, \sigma^{2}\right)$ refers to the complex 


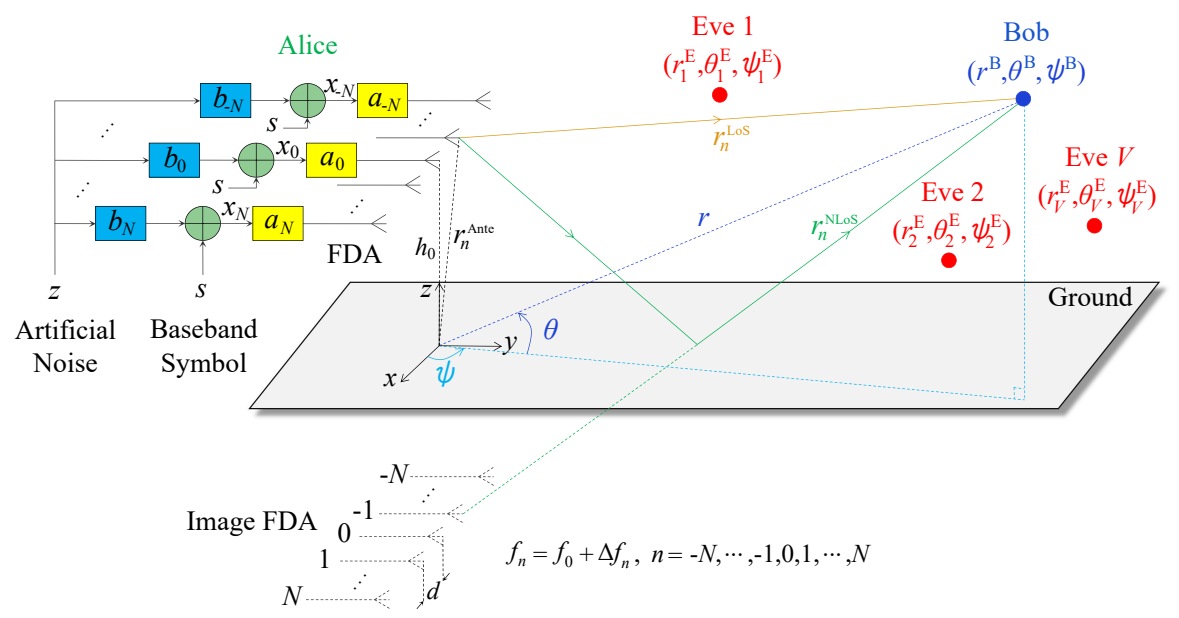

Fig. 1. The proposed two-ray multipath FDA-based DM model in MISOME wiretap channels.

Gaussian distribution with zero mean and variance $\sigma^{2}$. The unit vectors along the $x, y$ and $z$-axes of the Cartesian coordinate system are represented by $\overrightarrow{\mathbf{e}}_{x}, \overrightarrow{\mathbf{e}}_{y}$ and $\overrightarrow{\mathbf{e}}_{z}$, respectively. The expression, $\overrightarrow{\mathbf{r}}:=(r, \theta, \psi)$, refers to an arbitrary spatial point/vector $\overrightarrow{\mathbf{r}}$ with coordinates $(r, \theta, \psi)$ in the polar coordinate system. Additionally, $\overrightarrow{\mathbf{e}}_{r}$ is the unit vector of $\overrightarrow{\mathbf{r}}$.

\section{SySTEM MODEL}

As shown in Fig. 1, we consider an MISOME wiretap model where one transmitter (Alice) with multiple antennas is trying to transmit confidential information to one desired legitimate receiver (Bob), while $V$ anonymous passive eavesdroppers (Eves) are trying to intercept the confidential information.

Alice is composed of a symmetrical $(2 N+1)$-element FDA with equal element spacing $d$, which is set as half wavelength of the central carrier in this paper. These elements are linearly arrayed on the $x$-axis with the same height $h_{0}$ away from the ground. Thus, the position vector of the $n$-th $(n=-N, \cdots, 0, \cdots, N)$ element can be expressed as

$$
\overrightarrow{\mathbf{r}}_{n}^{\text {Ante }}=d_{n} \overrightarrow{\mathbf{e}}_{x}+h_{0} \overrightarrow{\mathbf{e}}_{z}
$$

where $d_{n}=n d$ is the $x$-axis coordinate of the $n$-th element.

The radiated frequency of the $n$-th element is designed as

$$
f_{n}=f_{0}+\Delta f_{n}=f_{0}+\Delta f \ln (|n|+1)^{g}
$$

where $f_{0}$ is the central radiated frequency, $\Delta f$ refers to a fixed frequency increment satisfying $|\Delta f| \ll f_{0}$, and $g$ represents an additional factor to control the frequency increments.

Let $\Omega$ denote the alphabet of the normalized baseband modulation symbols with size $|\Omega|=M$. Then the $n$-th antenna element of Alice's FDA is fed with the following signal,

$$
x_{n}=\sqrt{P_{s}}\left(\beta_{1} s+\beta_{2} b_{n} z\right)
$$

where $P_{s}$ indicates the total transmitting power; $s \in \Omega$ is the normalized baseband modulation symbol with $\mathbb{E}\left(|s|^{2}\right)=1$; $z \sim \mathcal{C N}(0,1)$ represents the circularly symmetric random complex Gaussian artificial noise (AN); $b_{n}$ denotes the complex weight coefficient of the AN feeding on the $n$-th element; $\beta_{1}$ and $\beta_{2}$ are power splitting factors for the useful signal and the AN, respectively, which satisfy the following constraint,

$$
\beta_{1}^{2}+\beta_{2}^{2}=1
$$

It is assumed that an arbitrary observation point is located at $\overrightarrow{\mathbf{r}}=r \overrightarrow{\mathbf{e}}_{r}:=(r, \theta, \psi)$, where $r, \theta$ and $\psi$ represent the distance from the coordinate origin to the observation point, the elevation and azimuth angles, respectively. In such a tworay multipath model, there exist two transmission paths for the signal transmitting from the $n$-th antenna element to the observation point. The first one is the direct line-of-sight (LoS) path $\overrightarrow{\mathbf{r}}_{n}^{\mathrm{LoS}}$, and the second is the reflected non-LoS (NLoS) path $\overrightarrow{\mathbf{r}}_{n}^{\mathrm{NLoS}}$. Geometrically, the length of the direct LoS path $\overrightarrow{\mathbf{r}}_{n}^{\mathrm{LoS}}$ can be calculated by [17]

$$
r_{n}^{\mathrm{LoS}} \approx r-\overrightarrow{\mathbf{e}}_{r} \cdot \overrightarrow{\mathbf{r}}_{n}^{\mathrm{Ante}}=r-d_{n} u-h_{0} v
$$

where $u \triangleq \cos \theta \cos \psi$ and $v \triangleq \sin \theta$.

For manipulable analysis, we suppose that the ground is an infinite and perfectly conducting plane so that the image theory is applicable. Therefore, the NLoS path can be equivalently regarded as an LoS path radiated from the image FDA, as shown in Fig. 1. This means that the length of the NLoS path from the $n$-the element to the observation point can be calculated by

$$
r_{n}^{\mathrm{NLoS}} \approx r-d_{n} u+h_{0} v
$$

For the sake of convenient analysis, we consider the normalized LoS channel in free space, and ignore the additive white Gaussian noise (AWGN), $\xi \sim \mathcal{C N}\left(0, \sigma_{\xi}^{2}\right)$. Then the received signal at an arbitrary observation point $\overrightarrow{\mathbf{r}}$ via the normalized LoS channel can be expressed in (7), where $a_{n}=e^{j \phi_{n}}$ is the excitation factor for the $n$-th element of the FDA.

For clarity, letting

$$
\left\{\begin{array}{l}
\mu_{n}=\exp \left\{j 2 \pi f_{0}\left(-\frac{r-d_{n} u}{c}\right)\right\} \\
\varepsilon_{n}=\exp \left\{j 2 \pi \Delta f_{n}\left(t-\frac{r-d_{n} u}{c}\right)\right\}
\end{array}\right.
$$




$$
\begin{aligned}
y^{\mathrm{LoS}}(\overrightarrow{\mathbf{r}}) & =\sum_{n=-N}^{N} a_{n} x_{n} \exp \left\{j 2 \pi f_{n}\left(t-\frac{r_{n}^{\mathrm{LoS}}}{c}\right)\right\}=\sum_{n=-N}^{N} a_{n} x_{n} \exp \left\{j 2 \pi f_{n}\left(t-\frac{r-d_{n} u-h_{0} v}{c}\right)\right\} \\
& =\sum_{n=-N}^{N} a_{n} x_{n} \exp \left\{j 2 \pi\left(f_{0}+\Delta f_{n}\right)\left(t-\frac{r-d_{n} u}{c}\right)\right\} \exp \left\{j \frac{2 \pi f_{n} h_{0} v}{c}\right\} \\
& =\exp \left\{j 2 \pi f_{0} t\right\} \sum_{n=-N}^{N} a_{n} x_{n} \exp \left\{j 2 \pi f_{0}\left(-\frac{r-d_{n} u}{c}\right)\right\} \exp \left\{j 2 \pi \Delta f_{n}\left(t-\frac{r-d_{n} u}{c}\right)\right\} \exp \left\{j \frac{2 \pi f_{n} h_{0} v}{c}\right\}
\end{aligned}
$$

TABLE I

One Possible Solution for $a_{n}$ AND $b_{n}$ SATISfying (15) with $N=3$

\begin{tabular}{cccccccc}
\hline$n$ & -3 & -2 & -1 & 0 & 1 & 2 & 3 \\
\hline$a_{n}$ & $0.998+0.050 j$ & $0.516+0.857 j$ & $0.384+0.923 j$ & $-0.955+0.296 j$ & $-0.996-0.091 j$ & $0.979-0.204 j$ & $0.222-0.975 j$ \\
$b_{n}$ & $0.286-0.958 j$ & $-0.938+0.346 j$ & $0.535-0.845 j$ & $-0.949+0.315 j$ & $-0.995+0.105 j$ & $-0.971-0.238 j$ & $-0.336+0.942 j$ \\
\hline
\end{tabular}

then the received LoS signal in (7c) will evolve into

$$
y^{\mathrm{LoS}}(\overrightarrow{\mathbf{r}})=\exp \left\{j 2 \pi f_{0} t\right\} \sum_{n=-N}^{N} a_{n} x_{n} \mu_{n} \varepsilon_{n} \exp \left\{j \frac{2 \pi f_{n} h_{0} v}{c}\right\}
$$

Similarly, the received signal at the observation point $\overrightarrow{\mathbf{r}}$ via the normalized NLoS channel can be written as

$$
\begin{gathered}
y^{\mathrm{NLoS}}(\overrightarrow{\mathbf{r}})=-\sum_{n=-N}^{N} a_{n} x_{n} \exp \left\{j 2 \pi f_{n}\left(t-\frac{r_{n}^{\mathrm{NLoS}}}{c}\right)\right\} \\
\approx-\sum_{n=-N}^{N} a_{n} x_{n} \exp \left\{j 2 \pi f_{n}\left(t-\frac{r-d_{n} u+h_{0} v}{c}\right)\right\} \\
=-\exp \left\{j 2 \pi f_{0} t\right\} \sum_{n=-N}^{N} a_{n} x_{n} \mu_{n} \varepsilon_{n} \exp \left\{-j \frac{2 \pi f_{n} h_{0} v}{c}\right\}
\end{gathered}
$$

The total received signal at the observation point $\overrightarrow{\mathbf{r}}$ can be obtained by adding the LoS signal in (9) and the NLoS signal in (10), which is expressed in (11). For clarity, we let

$$
\rho_{n}=j 2 \sin \left(\frac{2 \pi f_{n} h_{0} v}{c}\right)
$$

Then the total received signal in (11c) can be further simplified as (13), where

$$
\left\{\begin{array}{l}
\kappa \triangleq \sum_{n=-N}^{N} a_{n} \mu_{n} \varepsilon_{n} \rho_{n} \\
\eta \triangleq \sum_{n=-N}^{N} a_{n} b_{n} \mu_{n} \varepsilon_{n} \rho_{n}
\end{array}\right.
$$

In order to achieve a secure DM transmission from Alice to Bob, the next work is how to design the excitation factors of the FDA $\left\{a_{n}\right\}$ and the weight coefficients of the inserted AN $\left\{b_{n}\right\}$. By doing so, only Bob can acquire the useful information while Eves' received signals are severely distorted and interfered, thus providing transmission security between
Alice and Bob. We suppose that the location information of Bob, $\overrightarrow{\mathbf{r}}^{\mathrm{B}}:=\left(r^{\mathrm{B}}, \theta^{\mathrm{B}}, \psi^{\mathrm{B}}\right)$, is prior known for Alice, and then the design methodologies for $\left\{a_{n}\right\}$ and $\left\{b_{n}\right\}$ are given as

$$
\left\{\begin{array}{l}
\kappa^{\mathrm{B}}=\sum_{n=-N}^{N} a_{n} \mu_{n}^{\mathrm{B}} \varepsilon_{n}^{\mathrm{B}} \rho_{n}^{\mathrm{B}}=1 \\
\eta^{\mathrm{B}}=\sum_{n=-N}^{N} a_{n} b_{n} \mu_{n}^{\mathrm{B}} \varepsilon_{n}^{\mathrm{B}} \rho_{n}^{\mathrm{B}}=0
\end{array}\right.
$$

where $\mu_{n}^{\mathrm{B}}, \varepsilon_{n}^{\mathrm{B}}$, and $\rho_{n}^{\mathrm{B}}$ are calculated by substituting $(r, \theta, \psi)=$ $\left(r^{\mathrm{B}}, \theta^{\mathrm{B}}, \psi^{\mathrm{B}}\right)$ into (8) and (12).

Apparently, there are multiple solutions for (15), one of which is shown in Table I with $N=3$. If the solution sets of $\left\{a_{n}\right\}$ and $\left\{b_{n}\right\}$ are denoted by $\mathcal{A}$ and $\mathcal{B}$, respectively, the dynamic DM transmission can be achieved by dynamically and randomly generating $\left\{a_{n}\right\} \in \mathcal{A}$ and $\left\{b_{n}\right\} \in \mathcal{B}$ at symbol rate level. Once $\left\{a_{n}\right\}$ and $\left\{b_{n}\right\}$ are generated with the constraint of (15), the total received signal of Bob can be simplified into

$$
y^{\text {Total }}\left(\overrightarrow{\mathbf{r}}^{\mathrm{B}}\right)=\sqrt{P_{s}} \beta_{1} \kappa^{\mathrm{B}} s+\sqrt{P_{s}} \beta_{2} \eta^{\mathrm{B}} z=\sqrt{P_{s}} \beta_{1} s
$$

where only the useful signal is left while the inserted AN is eliminated. Therefore, Bob can easily recover the original confidential information from the received signal.

However, by contrast, the received signals of Eves at different locations from Bob will deteriorate seriously. Actually, on the one hand, the received useful signals of Eves are distorted owning to the non-accomplishment of the normalization property in (15); on the other hand, the received signals of Eves are severely interfered by the AN due to the failure of the orthogonal property in (15). Therefore, those Eves cannot intercept the confidential information, which guarantees the security of the confidential transmission from Alice to Bob.

\section{Performance Analysis}

BER and secrecy rate are widely used in DM-related literature to measure the performance of DM systems. The BER of DM systems was fully investigated in [18], which also 


$$
\begin{aligned}
& y^{\text {Total }}(\overrightarrow{\mathbf{r}})=y^{\operatorname{LoS}}(\overrightarrow{\mathbf{r}})+y^{\operatorname{NoS}}(\overrightarrow{\mathbf{r}}) \\
&=\exp \left\{j 2 \pi f_{0} t\right\} \sum_{n=-N}^{N} a_{n} x_{n} \mu_{n} \varepsilon_{n} \exp \left\{j \frac{2 \pi f_{n} h_{0} v}{c}\right\}-\exp \left\{j 2 \pi f_{0} t\right\} \sum_{n=-N}^{N} a_{n} x_{n} \mu_{n} \varepsilon_{n} \exp \left\{-j \frac{2 \pi f_{n} h_{0} v}{c}\right\} \\
&=\exp \left\{j 2 \pi f_{0} t\right\} \sum_{n=-N}^{N} j 2 a_{n} x_{n} \mu_{n} \varepsilon_{n} \sin \left(\frac{2 \pi f_{n} h_{0} v}{c}\right) \stackrel{\operatorname{LP}}{=} \sum_{n=-N}^{N} j 2 a_{n} x_{n} \mu_{n} \varepsilon_{n} \sin \left(\frac{2 \pi f_{n} h_{0} v}{c}\right) \\
& y^{\operatorname{Total}}(\overrightarrow{\mathbf{r}})= \\
&=\underbrace{\sum_{P_{s}}^{N} \beta_{1} s \sum_{n=-N}^{N} a_{n} x_{n} \mu_{n} \varepsilon_{n} \rho_{n}=}_{n=-N} \sum_{n=-N}^{N} a_{n} a_{n} \sqrt{P_{s}}\left(\beta_{1} s+\beta_{2} b_{n} z\right) \mu_{n} \varepsilon_{n} \rho_{n} \\
& \underbrace{\sqrt{P_{s}} \beta_{2} z}_{\text {Useful Signal }} \underbrace{\sum_{\text {Artificial Noise }}^{N} a_{n} b_{n} \mu_{n} \varepsilon_{n} \rho_{n}}_{n=-N} \triangleq \underbrace{\sqrt{P_{s}} \beta_{1} \kappa s}_{\text {Useful Signal }}+\underbrace{\sqrt{P_{s}} \beta_{2} \eta z}_{\text {Artificial Noise }}
\end{aligned}
$$

holds for the proposed two-ray multipath FDA-DM model. Here we will analyze the secrecy rate of the proposed two-ray multipath FDA-DM model.

When the total received signal in (13b) is polluted by the AWGN, $\xi \sim \mathcal{C N}\left(0, \sigma_{\xi}^{2}\right)$, the signal-to-noise ratio (SNR) $\gamma$ at an arbitrary observation point $\overrightarrow{\mathbf{r}}$ can be calculated by

$$
\gamma(\overrightarrow{\mathbf{r}})=\frac{P_{s} \beta_{1}^{2}|\kappa|^{2}}{\sigma_{\xi}^{2}}
$$

Correspondingly, the signal-to-interference-plus-noise ratio (SINR) $\lambda$ at the observation point $\overrightarrow{\mathbf{r}}$ can be calculated by

$$
\lambda(\overrightarrow{\mathbf{r}})=\frac{P_{s} \beta_{1}^{2}|\kappa|^{2}}{P_{s} \beta_{2}^{2}|\eta|^{2}+\sigma_{\xi}^{2}}
$$

Therefore, the achievable rate $\zeta$ at an arbitrary observation point $\overrightarrow{\mathbf{r}}$ can be written as

$$
\zeta(\overrightarrow{\mathbf{r}})=\log _{2}\{1+\lambda(\overrightarrow{\mathbf{r}})\}
$$

Especially, the achievable rate of Bob can be obtained by substituting (15) into (18) and (19), i.e.,

$$
\zeta\left(\overrightarrow{\mathbf{r}}^{\mathrm{B}}\right)=\log _{2}\left\{1+\frac{P_{s} \beta_{1}^{2}}{\sigma_{\xi}^{2}}\right\}
$$

We further presume that the $V$ eavesdroppers are located at $\overrightarrow{\mathbf{r}}_{\nu}^{\mathrm{E}}:=\left(r_{\nu}^{\mathrm{E}}, \theta_{\nu}^{\mathrm{E}}, \psi_{\nu}^{\mathrm{E}}\right)$, and they are different from Bob's location, which means $\overrightarrow{\mathbf{r}}_{\nu}^{\mathrm{E}} \neq \overrightarrow{\mathbf{r}}^{\mathrm{B}}$ for $\forall \nu \in\{1,2, \cdots, V\}$. Then, the achievable rate of the $\nu$-th Eve, $\zeta\left(\overrightarrow{\mathbf{r}}_{\nu}^{\mathrm{E}}\right)$, can be obtained by substituting $\overrightarrow{\mathbf{r}}=\overrightarrow{\mathbf{r}}_{\nu}^{\mathrm{E}}$ into (19).

Accordingly, the secrecy rate of the proposed two-ray multipath FDA-DM model, $\zeta_{\mathrm{sec}}$, can be calculated by

$$
\zeta_{\text {sec }}=\left[\min _{\nu \in\{1, \cdots, V\}}\left\{\zeta\left(\overrightarrow{\mathbf{r}}^{\mathrm{B}}\right)-\zeta\left(\overrightarrow{\mathbf{r}}_{\nu}^{\mathrm{E}}\right)\right\}\right]^{+}
$$

where $[*]^{+}=\max \{0, *\}$.

\section{Simulation Results}

The simulation parameters are selected as $f_{0}=10 \mathrm{GHz}$, $\Delta f=2 \mathrm{kHz}, g=1,\left(r^{\mathrm{B}}, \theta^{\mathrm{B}}, \psi^{\mathrm{B}}\right)=\left(150 \mathrm{~km}, 40^{\circ}, 70^{\circ}\right)$, $h_{0}=4.25 \lambda_{0}=4.25 c / f_{0}, P_{s}=1, \gamma=10 \mathrm{~dB}$, and $\pi / 4-$ QPSK, respectively. The locations of the passive Eves in our simulations are randomly generated.

Fig. 2(a)-(c) show the BER performances of the single-path and the proposed multipath FDA-based DM models versus range $r$, elevation angle $\theta$, and azimuth angle $\psi$, respectively. Two observations can be obtained from Fig. 2: a) The BER lobe of the proposed multipath model is more slim than the single-path model, which means that a more focusing secure area can be achieved via the proposed multipath model; b) As expected, larger $N$ and smaller $\beta_{1}$ can result in more slim BER lobe, which holds for both single-path and the proposed multipath models. Practically, in order to realize a more slim BER lobe, the number of array elements and the power splitting factor should be properly selected via weighing the array complexity and power efficiency.

The secrecy rate of the proposed two-ray multipath FDAbased DM model versus SNR (dB) is depicted in Fig. 3 (a). As expected, it shows that the secrecy rate of the proposed two-ray multipath FDA-based DM model increases with larger SNR, smaller $\beta_{1}$, and smaller number of Eves. Since the anonymous Eve may locate anywhere in free space, we further simulate the secrecy rates of the multipath and the single-path FDA-DM models versus Eve's location, as shown in Fig. 3 (b) and (c), respectively. Comparing Fig. 3 (b) and (c), it is again verified that the proposed multipath FDA-DM model can achieve more focusing secure area than the single-path model.

\section{CONCLUSION}

We investigated the ability of wireless physical layer secure transmission of the frequency diverse array-based directional modulation in multipath environments. By establishing the analytical methodology and conducting numerical simulations, 


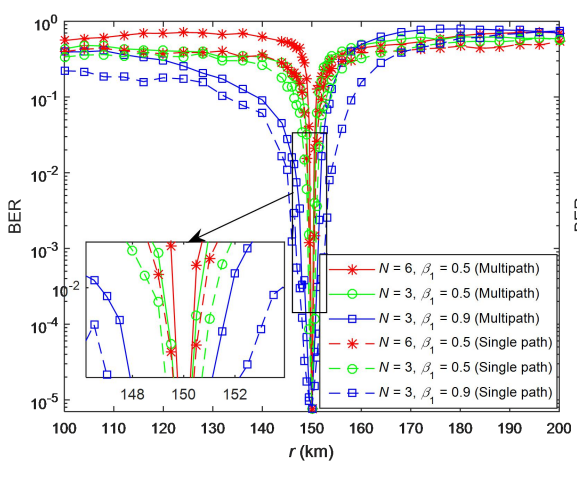

(a)

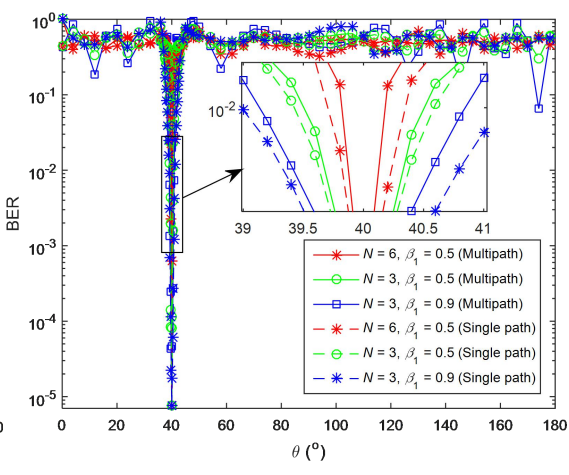

(b)

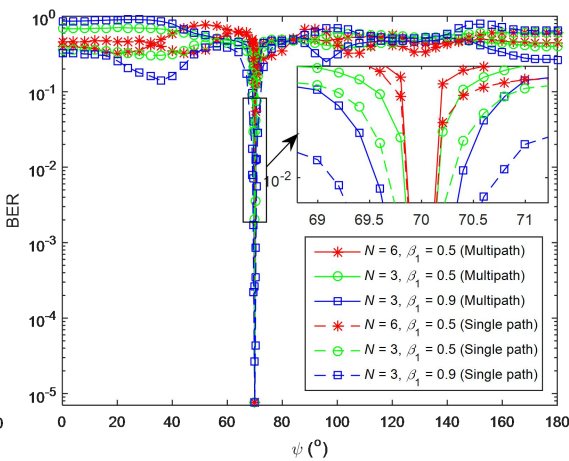

(c)

Fig. 2. BER performances versus (a) range $r$, (b) elevation angle $\theta$, and (c) azimuth angle $\psi$ for the single-path and the proposed multipath FDA-DM models.

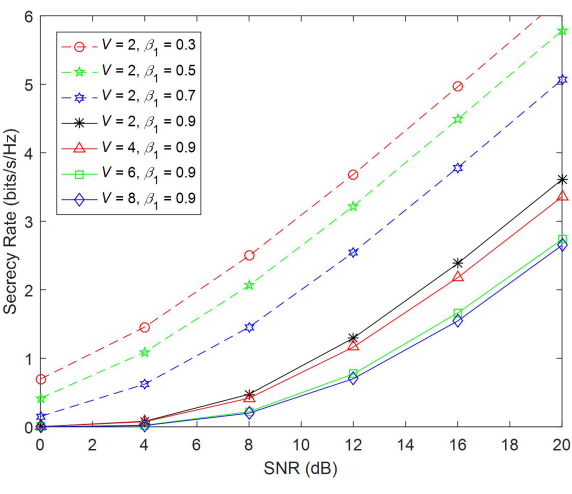

(a)

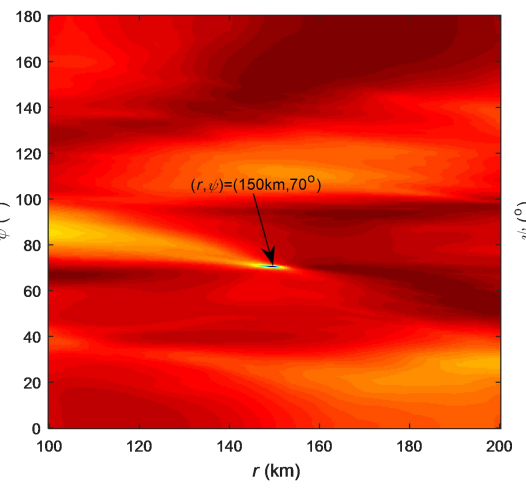

(b)

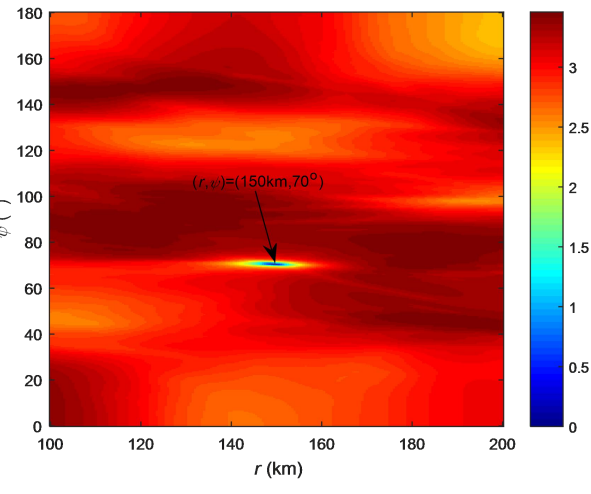

(c)

Fig. 3. Secrecy rate of the proposed multipath FDA-based DM model. (a) Secrecy rate versus SNR (dB); (b) Secrecy rate versus Eve's location (Multipath); (c) Secrecy rate versus Eve's location (Single path).

it was verified that the proposed FDA-based DM model is capable of wireless PLS transmission in two-ray multipath MISOME wiretap channels, which outperforms the single-path model in terms of more focusing secure area.

\section{REFERENCES}

[1] M. P. Daly and J. T. Bernhard, "Directional modulation technique for phased arrays," IEEE Tran. Ante. \& Prop., vol. 57, no. 9, pp. 2633-2640, Sep. 2009.

[2] Y. Ding and V. Fusco, "A review of directional modulation technology," International J. Microw. \& Wirel. Techn., vol. 8, no. 7, pp. 981-993, 2016.

[3] P. Antonik, M. C. Wicks, H. D. Griffiths, and C. J. Baker, "Frequency Diverse Array Radars," Proc. 2006 IEEE Radar Conf., 2006, pp. 215-217.

[4] W.-Q. Wang and H. C. So, "Transmit subaperturing for range and angle estimation in frequency diverse array radar," IEEE Trans. Signal Processing, vol. 62, no. 8, pp. 2000-2011, Apr. 2014.

[5] W. Khan, I. M. Qureshi, and S. Saeed, "Frequency diverse array radar with logarithmically increasing frequency offset," IEEE Ante. \& Wirel. Prop. Lett., vol. 14, pp. 499-502, 2015.

[6] H. Shao, J. Dai, J. Xiong, H. Chen, and W.-Q. Wang, "Dot-shaped rangeangle beampattern synthesis for frequency diverse array," IEEE Ante. \& Wirel. Prop. Lett., vol. 15, pp. 1703-1706, 2016.

[7] Y. Liu, H. Ruan, L. Wang, and A. Nehorai, "The random frequency diverse array: a new antenna structure for uncoupled direction-range indication in active sensing," IEEE J. Sel. Topics in Signal Processing, vol. 11, no. 2, pp. 295-308, Mar. 2017.

[8] J. Xiong, W.-Q. Wang, H. Shao, and H. Chen, "Frequency diverse array transmit beampattern optimization with genetic algorithm," IEEE Ante. \& Wirel. Prop. Lett., vol. 16, pp. 469-472, 2017.
[9] A. Basit, W. Khan, S. Khan, and I. M. Qureshi, "Development of frequency diverse array radar technology: a review," IET Radar, Sonar and Navigation, vol. 12, no. 2, pp. 165-175, Feb. 2018.

[10] J. Xiong, S. Y. Nusenu, and W.-Q. Wang, "Directional modulation using frequency diverse array for secure communications," Wirel. Personal Commun., vol. 95, no. 3, pp. 2679-2689, Aug. 2017.

[11] W.-Q. Wang, "DM using FDA antenna for secure transmission," IET Microw. Ante. \& Prop., vol. 11, no. 3, pp. 336-345, 2017.

[12] J. Hu, S. Yan, F. Shu, J. Wang, J. Li, and Y. Zhang, "Artificial-noiseaided secure transmission with directional modulation based on random frequency diverse arrays," IEEE Access, vol. 5, pp. 1658-1667, Jan. 2017.

[13] Q. Cheng, J. Zhu, T. Xie, J. Luo, and Z. Xu, "Time-invariant angle-range dependent directional modulation based on time-modulated frequency diverse arrays," IEEE Access, vol. 5, pp. 26279-26290, Nov. 2017.

[14] S. Ji, W.-Q. Wang, H. Chen, and Z. Zheng, "Secrecy capacity analysis of AN-aided FDA communication over Nakagami- $m$ fading," IEEE Wirel. Commun. Lett., vol. 7, no. 6, pp. 1034-1037, Dec. 2018.

[15] T. Xie, J. Zhu, Q. Cheng, and Y. Guan, "Secure point-to-multipoint communication using the spread spectrum assisted orthogonal frequency diverse array in free space," IEICE Trans. Commun., to be published. DOI: $10.1587 /$ transcom.2018EBP3314.

[16] J. Lin, Q. Li, J. Yang, H. Shao, and W.-Q. Wang, "Physical-layer security for proximal legitimate user and eavesdropper: a frequency diverse array beamforming approach," IEEE Trans. Inform. Forensics Security, vol. 13, no. 3, pp. 671-684, Mar. 2018.

[17] C. Cetintepe and S. Demir, "Multipath characteristics of frequency diverse arrays over a ground plane," IEEE Trans. Ante. \& Prop., vol. 62, no. 7, pp. 3567-3574, Jul. 2014.

[18] Y. Ding and V. Fusco, "Establishing metrics for assessing the performance of directional modulation systems," IEEE Trans. Ante. \& Prop., vol. 62, no. 5, pp. 2745-2755, May 2014. 